\title{
Les régimes territoriaux de l'économie sociale et solidaire : le cas du Pays Basque français
}

\author{
The territorial regimes of social and \\ solidarity-based economy : the case \\ of the French Basque Country
}

\author{
Xabier Itçaina \\ CNRS-SPIRIT, Sciences Po Bordeaux, Université de Bordeaux, \\ 11, allée Ausone, 33607 Pessac
}

\section{Résumé}

Par régime territorial de l'économie sociale et solidaire, on entend appréhender la construction de la confiance entre les acteurs locaux, les modalités de la régulation politique territoriale et la définition de problèmes publics. Cette perspective sera illustrée par les dynamiques de l'économie sociale en Pays Basque français, une mobilisation initiée par le mouvement coopératif dans les années 1970, étendue à de nouvelles dynamiques ensuite. Une fois retracées les modalités de la construction de la confiance, il s'agira de réfléchir à la part de l'économie sociale dans la construction politique du développement local, avant d'en observer la traduction dans les nouveaux réseaux de gouvernance qui accompagnent l'institutionnalisation du Pays Basque français à compter du milieu des années 1990.

(C) 2010 Lavoisier, Paris. Tous droits réservés.

\section{Summary}

When focusing on the «territorial regime » of the social and solidarity-based economy, this article puts under scrutiny the building of trust between the local actors, territorial political regulations and the definition of territorial public issues. This perspective will be exemplified by a case-study conducted on the social economy in the French Basque Country. This mobilisation was initiated by

*Adresse email : x.itcaina@sciencespobordeaux.fr

doi:10.3166/ges.12.71-87 @ 2010 Lavoisier, Paris. Tous droits réservés. 
the workers' cooperative movement in the mid-1970s, then extended to new dynamics. The article goes back to the process of trust-building, before analysing the role of social economy in the political construction of local development. It ends with a focus on the role played by social economy in the new governance networks emerging from the new institutionalization of the French Basque Country since the mid'1990s.

(c) 2010 Lavoisier, Paris. Tous droits réservés.

Mots clés : régime territorial, Pays Basque, économie sociale, gouvernance territoriale, coopératives, relations transfrontalières.

Keywords: territorial regime, French Basque country, social economy, worker's cooperatives, territorial governance, cross-border relations.

Réfléchir ${ }^{1}$ aux interactions entre économie sociale et solidaire (ESS dans le reste du texte), développement local et institutionnalisation territoriale relève de la double gageure. Une telle démarche implique d'une part le repérage de dynamiques territoriales suffisamment homogènes pour que l'on puisse en observer l'évolution dans sa profondeur historique. Parler d'ESS, ensuite, suppose que l'on constitue en champ cohérent un ensemble d'initiatives et de formes organisationnelles reliées entre elles par l'adhésion à des valeurs et à des principes d'action les situant sur une position médiane entre logiques purement marchandes et sphère de la redistribution publique. D'avantage qu'un secteur économique ou d'action publique stabilisé, l'ESS constitue plutôt un champ de pratiques et de représentations dont le bornage constitue en soi un enjeu majeur.

Le Pays Basque français ${ }^{2}$ fournit, à ce titre, un bon ancrage empirique pour l'observation croisée des dynamiques de développement territorial et d'ESS. L'objectif de cet article consiste à réfléchir aux articulations entre deux dynamiques souvent pensées séparément. D'une part, ce territoire est porteur de dynamiques d'ESS qui lui confèrent une spécificité à l'échelle régionale, une mobilisation initiée par un mouvement de création de coopératives de production à partir des années 1970, puis étendu à de nouvelles dynamiques solidaires dans les années 1990 (microfinance, commerce équitable, associations culturelles et linguistiques, développement local, agriculture paysanne). Sur le plan territorial ensuite, le Pays Basque français a expérimenté depuis la fin des années 1980 un processus d'institutionnalisation territoriale issu d'une démarche participative originale, et ayant conduit à la constitution d'un « pays », en son temps une innovation à l'échelle française, et d'un tissu d'institutions territoriales sectorielles et généralistes.

Ce texte s'interroge sur l'articulation de ces deux dynamiques. Sur le plan théorique, il revient à se demander en quoi leur croisement contribue à définir un régime territorial de l'ESS spécifique au Pays Basque. L'approche en termes de régime territorial permet d'appréhender les mutations du développement local et de l'action publique à trois échelles : la construction de la confiance entre les acteurs, les modalités de la régulation politique

\footnotetext{
${ }^{1}$ Je remercie les membres du groupe de travail CIRIEC-France et les deux évaluateurs de la revue pour leurs lectures critiques.

${ }^{2}$ Les trois provinces d'Ancien régime basque françaises (Labourd, Basse-Navarre, Soule) constituent, avec le Béarn, le département des Pyrénées-Atlantiques.
} 
systémique et la définition de problèmes publics (Itçaina et Palard 2007). Par l'analyse de la confiance, on entend analyser les facteurs de constitutions d'un capital social territorial qui constitue, en tant que bien collectif, un facteur de production à part entière (Trigilia 2002 , p. 201). Par régulation politique systémique, l'on désigne les formes de la gouvernance d'un territoire qui caractérisent une structure composée par différents acteurs et institutions permettant d'apprécier les règles et routines qui donnent sa spécificité à un lieu et de penser les rapports qui s'établissent entre système économique de production et système politique. Enfin, par l'attention portée à la construction des problèmes publics sur le territoire, l'on s'interroge sur la transformation d'enjeux sociaux en problèmes publics, puis en problèmes politiques (Lascoumes et Le Galès 2007). Volontiers généraliste, cette approche offre une perspective en amont et en aval des dynamiques territoriales, depuis la constitution sociale de la confiance jusqu'aux instruments d'action publique. Elle aide à fonder l'hypothèse qui guidera ce texte, selon laquelle la trajectoire historique de l'ESS en Pays Basque depuis les années 1970 est totalement imbriquée dans les mobilisations sociopolitiques territoriales. En ce sens, l'ESS basque, et singulièrement le mouvement coopératif, a évolué d'une genèse militante vers une amorce d'institutionnalisation correspondant à la structuration d'une gouvernance territoriale originale dans les années 1990-2000. Cette évolution sera retracée ici en trois temps. Une fois avoir retracé les modalités de la construction sociale de la confiance sur ce territoire, il s'agira de réfléchir au rôle de l'ESS dans la construction politique du développement local, avant d'en observer la traduction dans les nouveaux instruments de la gouvernance territoriale.

\section{La construction sociale de la confiance}

Matrice territoriale, capital social territorial, qualités du territoire, réseaux sociaux, dispositions à coopérer : comment analyser ce socle économique, social et culturel qui sert de base à la construction des réseaux territoriaux ayant vocation à donner naissance aux expériences d'ESS ? La genèse de ces réseaux doit être relue à la lumière d'une construction sociale de la confiance territoriale qui repose sur un équilibre entre dispositions héritées et démarches stratégiques. La structuration de l'ESS basque se manifeste d'abord par un mouvement de création de SCOP (sociétés coopératives ouvrières de production) à compter de 1975, qui singularisera durablement le Pays Basque à l'échelle régionale. Elle résulte d'une alliance originale entre un fort degré de confiance communautaire (appartenance territoriale basque) et de confiance organisationnelle (le choix de la forme coopérative) (Dupuy et Torre, 2004) ${ }^{3}$. L'ancrage organisationnel permettra aux promoteurs du coopérativisme d'accéder à de nouveaux réseaux et de s'extraire des seules affiliations communautaires, qui pouvaient avoir un effet économique contre productif en focalisant la réflexion sur un mode de développement purement endogène et orienté vers les réseaux et les marchés locaux. Entre autres facteurs, l'on soulignera ici l'importance de quatre

\footnotetext{
${ }^{3}$ Dupuy et Torre (2004) distinguent la confiance interpersonnelle, où la relation se limite à un cadre interindividuel, où la confiance résulte du caractère répété des transactions, d'une recherche individuelle de gains et d'une rationalité marchande. En matière de confiance communautaire en revanche, toute relation humaine est encastrée dans des réseaux sociaux, la relation de confiance s'appuie sur une réputation inscrite dans ces réseaux, la rationalité marchande n'explique pas tous les comportements économiques. Enfin, la confiance organisationnelle constitue une extension des principes de la confiance interpersonnelle à l'action collective.
} 
facteurs d'ordre religieux, culturel, politique et économique dans la structuration d'une atmosphère territoriale propice à l'émergence d'une dynamique d'économie sociale.

Le catholicisme, à la fois comme horizon éthique et comme réseau d'institutions quadrillant la société, a constitué un élément de contexte favorable à l'éclosion d'un mouvement coopératif (Itçaina, 2007b). J. Palard et Thierry Berthet ont souligné, dans l'étude sur le développement industriel du bocage vendéen, l'importance du «fragment » religieux (paroisse, enseignement privé, Jeunesse agricole chrétienne) comme porteuse d'une conception communautaire du développement et de rapports privilégiés entre éthique catholique et d'un esprit local du capitalisme, sans forcément d'ailleurs que ces rapports débouchent sur une dynamique d'économie sociale proprement dite (Berthet et Palard 1997). En Pays Basque, sur le plan éthique, le coopérativisme a pu être adopté par des prêtres et des militants catholiques en recherche d'une voie médiane entre socialisme et individualisme libéral. Côté espagnol, le complexe coopératif de Mondragón a été fondé dans les années 1940-1950 par un vicaire catholique, secondé par un groupe de l'Action catholique ouvrier, sensible notamment aux thèses personnalistes et porteur d'une pensée innovante sur le travail (Azurmendi, 1984). Côté français, des prêtres sensibilisés à la question sociale, des groupes d'Action catholique spécialisée, de jeunes ingénieurs chrétiens contribueront à diffuser l'idée coopérative et, singulièrement, le modèle de Mondragón dans les années 1960, auprès de milieux sociaux propices à initier à leur tour une dynamique coopérative.

On rangerait plutôt du côté des « logiques sociales non intentionnelles $»^{4}$ ou, plus exactement, des représentations, une caractéristique mise en avant par les premiers coopérateurs, celle des pratiques coutumières d'entraide et de réciprocité propres au milieu rural. Invoquées également au titre de contexte culturel, voire de précédent structurel, les pratiques réciprocitaires fondées sur le droit coutumier ont pu constituer l'un des terreaux, en diffusant la représentation d'une société supposée égalitariste, au coopérativisme. Plusieurs fondateurs de la première vague des SCOP, en particulier, mettent en avant des parallèles structurants : Le caractère indivisible de la «maison-exploitation » se retrouverait dans les réserves impartageables des coopératives, la dimension collective du travail entre voisins (auzolan) dans la dimension égalitaire de la coopération, la démocratie délibérative coutumière dans la gouvernance des coopératives (Itçaina, 2007b). Ces lectures a posteriori insufflent de la cohérence en lissant la relecture des modèles hérités. Des relations sociales fortement communautaires, comme dans la société rurale, ne sont pas automatiquement favorables à la coopération. Les travaux d'anthropologie juridique et de sociologie historique du milieu rural basque ont souligné, depuis longtemps déjà (Bidart, 1977), que les relations entre les maisons relevaient au moins autant d'un « individualisme familial » que de dispositifs réciprocitaires, que l'indivisibilité du patrimoine familial reposait sur un régime successoral inégalitaire (droit d'aînesse absolu), qu'il a toujours existé une hiérarchie entre maisons selon le régime de propriété et la position sociale. Reste que la représentation d'un imaginaire économique égalitariste a pu jouer un rôle dans les motivations des fondateurs des premières coopératives.

\footnotetext{
${ }^{4}$ Une lecture en termes de logiques sociales non intentionnelles donnerait la primeur aux propriétés structurelles du territoire, qui constitueraient un cadre d'action défini a priori dans lequel s'inscriraient les stratégies des acteurs. Une telle posture a pu être appliquée par J. Palard à « l'énigme » du développement industriel de la région québécoise de la Beauce (Palard 2009).
} 
L'accent mis sur ces logiques non intentionnelles ou sur les propriétés du territoire est insuffisant pour expliquer l'émergence des dynamiques d'économie sociale. L'analyse ne peut faire l'économie d'une approche stratégique centrée sur les acteurs et l'action collective. Les cultures politiques en présence permettent d'expliquer les conditions d'émergence historique des entreprises associatives. Le lien complexe et historiquement heurté entre catholicisme, nationalisme et coopérativisme est bien connu pour le Pays Basque espagnol, à condition toutefois de ne pas faire l'impasse sur la part du mouvement ouvrier basque dans la genèse des premières coopératives de consommation et de production au début du $\mathrm{XX}^{\mathrm{e}}$ siècle Cette part des cultures politiques a été moins considérée côté basque français. Or, l'émergence des coopératives de production à partir du milieu des années 1970 est fortement liée à un mouvement nationaliste basque, qui se sécularise et prend un tournant autogestionnaire au début des années 1970. L'économie sociale constitue alors le bras économique d'une mobilisation politique à plus large surface sociale.

L'émergence d'une dynamique originale d'économie sociale s'explique ainsi par l'alliance conjoncturelle de propriétés structurelles du territoire (catholicisme social et héritages réciprocitaires), de représentations du monde et de stratégies politiques aspirant à allier mobilisation identitaire et principes coopératifs. S'y greffe un contexte de crise économique qui, à partir du milieu des années 1970, constituera sans doute l'accélérateur de l'implantation du mouvement coopératif, en Pays Basque comme ailleurs en France (Demoustier, 2001). À l'instar de l'expérience coopérative de Mondragón qui émerge au lendemain de la guerre civile sur un territoire, le Guipuzcoa, fortement éprouvé et en proie à des difficultés socio-économiques considérables, le mouvement coopératif basque français émerge dans un contexte de forte incertitude économique propice aux innovations propres aux entreprises collectives. Les SCOP basques s'adapteront ensuite de façon diverse aux pressions du marché, au prix parfois d'une atténuation du marquage en valeurs originel (Itçaina, 2007a).

L'insistance sur l'ensemble de ces dimensions - y compris le rôle d'accélérateur de la crise - aspire ainsi à éviter le malaise que peut légitimement susciter, en particulier chez les économistes (Lung 2007), la prise en compte de la dimension matricielle des dynamiques territoriales. Certains auteurs ont eu recours à la notion de capital social pour qualifier ces dispositions à coopérer, qui n'est pas sans lien avec l'ESS dans la mesure ce capital social territorial se repère souvent au travers du degré de participation aux associations volontaires, dans toutes leurs variantes (dans l'approche la plus extensive : tout ce qui n'est ni État, ni marché, ni famille) (Newton 1999). Souvent insuffisamment contextualisées, les approches en termes de capital social conduisent parfois à des corrélations fragiles entre confiance sociale et confiance dans les institutions, ou tendent à assimiler capital social et capital politique (Ritaine 2001). On parlerait plutôt dans le cas basque d'un territoire à fort «capital civique » au sens d'A. Evers', afin d'insister l'imbrication sociale et proprement politique de ces initiatives économiques.

\footnotetext{
5 "Social capital is then to be understood both as an indicator of the degree of development of a civic society (constituted both by social and political action) and a way to debate civic commitment with an eye on economic development and governance." (Evers, 2001, p. 299).
} 


\section{2. Économie sociale et politisation du développement local}

Le développement local et l'économie sociale font l'objet dans les années 1970-1980, d'un processus de politisation en tant que « requalification des activités sociales les plus diverses, requalification qui résulte d'un accord pratique entre des agents sociaux enclins, pour de multiples raisons, à transgresser ou à remettre en cause la différenciation des espaces d'activité. » (Lagroye 2003, p. 361). La politisation dont font l'objet les expériences entrepreneuriales se manifeste au travers de trois dimensions : la politisation de l'enjeu du développement local, le choix de la formule coopérative, et l'articulation avec les dynamiques transfrontalières.

Le développement local, à compter du milieu des années 1960, constitue une nouvelle priorité pour des milieux militants nationalistes et régionalistes basques jusque là essentiellement centrés sur des revendications institutionnelles, linguistiques et culturelles. En ce sens, cette nouvelle préoccupation pour le développement local reprend, en la radicalisant, l'héritage démocrate-chrétien qui, dans l'après-guerre, contribue en particulier à la modernisation de l'agriculture basque. Dans les années 1960, le nationalisme basque se distancie progressivement de cet héritage en s'ancrant plus clairement à gauche, à l'instar de bien d'autres mouvements régionalistes en France. Le développement local, de fait, est construit par le discours militant comme un problème public (Lascoumes et Le Galès, 2007), et le mouvement des SCOP basques s'érige alors en contestation potentielle de l'ordre institutionnel local. S'articule ainsi un discours doublement contestataire à l'encontre, d'abord, de l'inertie supposée des pouvoirs publics et des élites locales et nationales, mais également contre un mouvement basque ayant négligé la question économique. L'idée d'un développement économique endogène s'expose tout au long des années 1960 et 1970 dans les journaux nationalistes, comme l'hebdomadaire Enbata, ou dans la presse bascophone de sensibilité démocrate-chrétienne, comme Herria. Longtemps soutenue par une fraction militante du clergé, cette revendication se sécularise progressivement et est portée en particulier par de jeunes enseignants issus de l'enseignement technique et agricole à Hasparren ou Saint-Palais. À la fin des années 1970, l'économie sociale et le mouvement coopératif sont au cœur de cette mise en politique, en généralisant la représentation du caractère indissociable d'un développement économique local endogène et de la construction politique du territoire. Cette montée en généralité se traduit, au-delà du seul mouvement des SCOP, par l'émergence de diverses initiatives : association de développement local Hemen en 1979, qui deviendra un instrument majeur de promotion de l'économie sociale et solidaire, société de capital-risque Herrikoa en 1980, qui ambitionne de constituer l'outil financier indispensable à l'innovation économique locale, structures de microcrédit plus récemment, etc. Enfin, loin d'être monopolisée par le nationalisme, l'idée d'une prise en charge concertée d'un développement local à multiples facettes sera également portée par des acteurs proches de la Chambre de commerce et d'industrie de Bayonne, et par les acteurs du système politico-administratif local. Le CELIB ${ }^{6}$ breton inspirera fortement ces démarches qui, en matière d'action publique, se traduisent, dès le milieu des années 1970 , par des procédures de Contrat de pays en Soule, puis des démarches similaires en pays de Mixe et d'Ostabarret en Basse-Navarre.

\footnotetext{
${ }^{6}$ Comité d'études et de liaison des intérêts bretons.
} 
La politisation passe également par la promotion de l'économie sociale, et en particulier de la forme coopérative, comme réponse organisationnelle potentielle aux carences du développement local. Certes, loin d'être monopolisée par l'économie sociale, la référence à l'identité territoriale est également portée par le secteur capitaliste classique. Dans une étude récente sur les motivations des chefs d'entreprise d'Aquitaine, F. Reix souligne l'attachement au territoire chez les entrepreneurs originaires du Pays Basque, qu'ils relèvent de l'économie sociale - comme ce créateur d'une SCIC (Société coopérative d'intérêt collectif) de la région bordelaise - ou pas (Reix 2008). Reste que le statut coopératif, par la charge politique qu'il contient, insuffle une spécificité à la démarche entrepreneuriale. Dans les années 1950-1970, l'expérience sudiste de Mondragón est perçue comme un exemple vertueux en matière de coopérativisme intégré associant système productif industriel, banque, consommation et formation. Les tentatives d'adoption d'un coopérativisme intégral en milieu agricole, comme l'expérience, alors internationalement connue, du village de Zuñiga en Navarre espagnole, font également l'objet d'une attention soutenue côté français. La coopérative de Zuñiga, soulignera-t-on alors, a été créée par un prêtre, «par des Basques et pour des Basques », et elle s'éloigne du modèle communiste de la collectivisation (Herria, 1967). Dans les années 1970, l'inspiration du catholicisme social s'effilochera progressivement au profit d'une empreinte plus prononcée du mouvement coopératif français et international. La firme coopérative est alors perçue comme une alternative fondée sur des principes hautement politiques : démocratie interne, échelle limitée de salaires, primauté du travail sur le capital, participation aux bénéfices, réserves impartageables, ancrage territorial. Cette pensée innerve la création en 1974 d'une association, Partzuer (associé), destinée à impulser la création du coopérativisme en Pays Basque français, et le développement des firmes à partir de 1975. Un tel ancrage dans l'économie sociale permet ainsi aux fondateurs des SCOP basques de bénéficier du soutien du mouvement coopératif à l'échelle française et, incidemment, des politiques publiques y afférant. Le mouvement profite ainsi des politiques incitatives à la fin des années 1970 (notamment de la loi sur les SCOP de 1978) et du début d'institutionnalisation gouvernementale de l'économie sociale à partir de 1981 (Demoustier 2001).

Un troisième facteur de politisation provient du rôle joué par le Pays Basque espagnol dans la structuration de ces mobilisations côté français. À tort ou à raison, la circulation transfrontalière des expériences, des expertises et des acteurs renforce, notamment chez les détracteurs du mouvement coopératif, l'impression d'imbrication transfrontalière des enjeux économiques et politiques. Or si échange il y a, il ne conduit pas forcément à de la politisation. L'influence du modèle de Mondragón dans la genèse du mouvement coopératif basque français a déjà été soulignée. Elle se traduira, au-delà des voyages d'études qualifiés a posteriori de "pèlerinages coopératifs » par les acteurs, par un soutien tangible. Plusieurs futurs fondateurs de SCOP effectuent des séjours à Mondragón à la fin des années 1970, parfois jusqu'à deux années, où ils reçoivent à la fois une formation technique en milieu industriel, et une socialisation politique au coopérativisme ${ }^{7}$. Mondragón s'impliquera également fortement dans la naissance d'une coopérative de meubles à Saint-Martin-d'Arrossa en 1979. Plusieurs réfugiés ayant fui le versant espagnol pour des raisons politiques trouvent dans le mouvement coopératif basque fran-

\footnotetext{
${ }^{7}$ Entretien, dirigeant d'une SCOP de meubles, Itxassou.
} 
çais l'opportunité de faire valoir un triple savoir-faire, celle d'un mouvement coopératif sudiste autrement puissant et structuré, celle d'une compétence technique entrepreneuriale acquise en milieu industriel et celle d'une politisation de la question basque plus radicale dans ses moyens et dans ses conséquences. Cette impulsion transfrontalière, décisive aux origines du mouvement, ne se stabilisera sur la longue durée que pour les partenariats économiquement viables pour les deux parties.

La conjugaison de ces trois facteurs contribue à construire le développement local comme un problème public, en générant des controverses, puis comme un problème politique lorsque la mobilisation parvient « à acquérir une capacité d'interpellation telle que des acteurs qui n'étaient pas initialement concernés par l'enjeu prennent des positions et argumentent en sa faveur ou contre lui » (Lascoumes et Le Galès 2007 : 80). Les coopérateurs militants des années 1970-1980 sont essentiellement des jeunes, pas ou peu qualifiés, à l'exception de quelques ingénieurs et enseignants. Ces acteurs produisent un discours de légitimation cherchant à justifier la primeur de la formule coopérative en matière de développement local. Stone (1989) distingue quatre composantes des discours de légitimation dans la construction d'un problème public, que l'examen de la production du mouvement coopératif basque (presse, archives de Lana ${ }^{8}$, entretiens auprès des fondateurs des SCOP) permet d'illustrer. Le discours de légitimation se fonde tout d'abord sur une explication analytique de la situation : ici, le raisonnement se fonde sur une lecture en termes de sousdéveloppement économique du Pays Basque français, que l'on perçoit alors comme partagé entre une agriculture déclinante, un tissu industriel en crise, un développement basé sur le tourisme et les services. L'alternative est celle d'un développement endogène par la petite et la moyenne industrie, en cherchant l'équilibre entre la côte basque et l'intérieur des terres. Le cas exemplaire est fourni par l'exemple omniprésent de Mondragón. Les métaphores reliant les enjeux sont fréquentes : développement économique et culturel, démocratie dans l'entreprise et démocratie politique, contestation économique et contestation politique. Enfin, l'ambiguitté de certains arguments établissant des significations multiples est tout aussi sensible, notamment dans les débats qu'a pu générer la dimension identitaire de l'expérience basque au sein du mouvement coopératif à l'échelle française.

L'économie sociale basque, essentiellement coopérative dans les années 1970-1980, se pense alors en mouvement, voire en mouvement social (Itçaina, 2007a). À compter du milieu des années 1980, quelques déconvenues entrepreneuriales et la réorientation des politiques nationales en direction du secteur associatif (Demoustier, 2001, p. 54-55) ralentiront les créations de SCOP. L'économie sociale et solidaire basque continue néanmoins de se développer en se diversifiant, tant d'un point de vue statutaire que sectoriel (commerce équitable, microfinance ${ }^{9}$, agriculture paysanne, insertion, etc.). De fait, et à l'exception notable des $\mathrm{SCOP}^{10} \mathrm{et}$

\footnotetext{
${ }^{8}$ L'association Lana (travail/emploi), très active dans les années 1980, ambitionne de fédérer les différentes expériences coopératives basques au sein d'une organisation intégrée, à l'image de Mondragón.

${ }^{9}$ Brana et Jégourel (2010) ont souligné la spécificité du Pays Basque, et notamment de la Soule, dans le paysage de la microfinance aquitaine.

${ }^{10}$ En 2007, les Pyrénées-Atlantiques restent le premier département pour le nombre de SCOP en Aquitaine (33 sur 92, dont 24 en Pays Basque et 9 en Béarn) et le nombre de salariés des SCOP (577 sur 1402, dont 404 en Pays Basque et 173 en Béarn). Ces PME (entre 2 et 83 salariés) déploient leurs activités dans plusieurs secteurs : imprimerie, électronique, lignes téléphoniques et électriques, équipement automobile, meubles, services (données URSCOP Aquitaine).
} 
d'une dynamique associative prononcée, l'ESS basque ne présente pas, dans les années 2000, de grande spécificité statistiquement repérable à l'échelle régionale. En 2007, le nombre d'emplois équivalents temps plein dans l'ESS basque - hors agriculture - est légèrement supérieur (10,95\% de l'emploi total) en Pays Basque qu'à l'échelle aquitaine en $2006(9,7 \%)^{11}$. La part dans l'ensemble de l'économie locale des établissements relevant de l'ESS est sensiblement identique en Pays Basque (9,71\% en 2007) et en Aquitaine (9,6\% en 2006). Enfin, tout comme en Aquitaine, la féminisation des emplois est largement supérieure dans l'ESS basque (69\% du total des postes) que dans l'ensemble de l'économie locale $(49 \%)^{12}$. En d'autres termes, si spécificité de l'ESS basque il y a, elle ne se laisse pas forcément saisir par la statistique, mais davantage au détour de processus historique qui font s'entremêler des logiques solidaires, territoriales et proprement politiques. Ce mélange connaitra une nouvelle déclinaison avec le changement des formes de la gouvernance territoriale.

\section{3. Économie sociale et solidaire et nouvelle gouvernance territoriale}

On ne saurait conclure, à la lecture de ce qui précède, à un processus exclusivement centré sur la société civile et uniquement pensé comme alternative à l'action publique. En l'occurrence, il s'agit de se demander si le tournant dans la gouvernance territoriale qu'expérimente le Pays Basque à partir de la fin des années 1980 constitue véritablement une fenêtre d'opportunité pour une inscription de l'ESS sur le nouvel agenda de la gouvernance locale. Pour répondre à cette question, il faut, avant de parler d'ESS, dresser à grands traits les caractéristiques du changement que connait la gouvernance territoriale en Pays Basque. L'on s'interrogera ensuite sur la façon dont il est possible de repérer le rôle des organisations d'ESS dans ce processus, avant d'illustrer le propos par un exemple sectoriel particulièrement pertinent en Pays Basque, celui des mobilisations linguistiques et culturelles.

\subsection{Le contexte: une nouvelle gouvernance territoriale en Pays Basque}

Les transformations de la régulation publique du développement local dans les années 1990 modifient la donne. D'un rapport distancié, voire conflictuel, à la puissance publique, les acteurs de l'économie sociale évoluent vers des postures plus partenariales, en accompagnant la nouvelle gouvernance du territoire, au sens d'un processus « de coordination des acteurs, mais aussi d'appropriation des ressources et de construction de la territorialité » (Leloup, Moyart et al. 2005). L'institutionnalisation territoriale franchit en effet une nouvelle étape en Pays Basque à partir de la fin des années 1980. Elle prendra la forme d'une démarche participative et prospective, à l'initiative de l'État qui entend ainsi retrouver à la fois son rôle d'animateur sur ce territoire réputé réfractaire, et répondre également à la revendication d'une individuation institutionnelle Pays Basque, exprimée par bon nombre d'élus et de représentants de la société civile.

\footnotetext{
${ }^{11}$ Ces données ont été obtenues en croisant les sources INSEE-CLAP 2007 pour les bassins d'emplois Bayonne-Pyrénées et Communauté de communes de Soule d'une part, et les synthèses régionales de la CRESS Aquitaine d'autre part (CRESS, 2008). Je remercie la CNCRESS, la DIIESES et la CRESS Aquitaine pour l'accès à ces données.

${ }^{12}$ Cette primauté féminine se retrouve dans les associations et les mutuelles, mais pas dans les coopératives.
} 
Une série de concertations entre représentants de la société civile, acteurs politiques et économiques, « développeurs » s'articule ainsi à la fin des années 1980 autour d'une démarche prospective dite « Pays Basque 2010 ». Cette procédure de concertation généralisée se traduira par la création d'un Institut culturel basque en 1989, d'un Conseil de développement du Pays Basque, associant acteurs de la société civile, acteurs socio-économiques et politiques, et d'un Conseil des élus du Pays Basque en 1994-1995³. En 1997, le territoire est reconnu comme un « pays » au sens de la loi de 1995. Le travail de ces institutions débouchera sur la signature en 1997 d'une première Convention de développement avec l'État, puis en 2000 d'une Convention spécifique pour 2001-2006, marquant l'engagement de l'État et des collectivités locales en faveur du développement local. La démarche connaît un renouveau au début des années 2000, avec une nouvelle démarche prospective Pays Basque 2020 à partir de 2005, qui débouche en septembre 2008 sur un nouveau contrat territorial à l'échelle du Pays Basque. Se dessine ainsi une nouvelle forme de gouvernance territoriale à deux dimensions. D'une part, l'association étroite au processus des acteurs issus de la société civile fait de l'expérience basque un laboratoire de la nouvelle gouvernance territoriale, au sens où s'y déploient « des situations de coopération non ordonnées par la hiérarchie qui correspondent à la construction, à la gestion ou à la représentation de territoires, en particulier face à leur environnement économique ou institutionnel. » (Simoulin 2007). D'autre part, ces procédures participatives débouchent sur des processus d'institutionnalisation, au sens où « les règles et les procédures sont de plus en plus nombreuses, gagnent en précision et deviennent réellement prescriptives » (Lascoumes et Le Galès, 2007 : 98). Loin de constituer de simples institutions consultatives, les nouvelles instances, par les démarches contractuelles avec l'État et les collectivités locales qu'elles génèrent, contribuent en effet à instaurer de nouvelles règles et procédures, et surtout à banaliser le Pays Basque comme cadre territorial d'action publique ${ }^{14}$.

Une nouvelle communauté de politique publique se constitue en Pays Basque autour du développement territorial. Ces communautés rassemblent des acteurs présentant trois caractéristiques : ils disposent de compétences et d'intérêts spécifiques sur un domaine d'action publique ; ils appartiennent à des milieux de nature différente : administrative, économique, universitaire, médiatique, politique, associative, etc. ; ils ont un fort degré d'engagement sur un enjeu, un rôle actif aux différentes phases de la définition du problème concerné, des choix de politique publique et de suivi de la mise en œuvre (Lascoumes et Le Galès, 2007). Autant de caractéristiques réunies dans la «nouvelle grammaire du territoire » (Ségas 2004) qui se met progressivement en place en Pays Basque, grammaire qui doit autant à l'installation de la gouvernance comme nouveau paradigme d'action publique qu'au précédent conflictuel lié au mouvement identitaire (Chaussier 1996). La traduction par l'expertise du développement territorial entend également, en la technicisant, contribuer à dépolitiser un enjeu polarisé autour de tensions identitaires. La communauté de politique publique qui se fonde autour de la gouvernance territoriale basque peut être ainsi analysée comme l'alliance, au sens de Bernard

\footnotetext{
${ }^{13}$ Si le Conseil de développement du Pays Basque a été installé en 1994, c'est la loi Voynet de 1999 qui a généralisé à tous les pays ce type de dispositif et le cadre de développement territorial institué dès la loi Pasqua de 1995. L'expérience basque aurait largement inspiré cette généralisation (Hourcade, 2007, p. 7).

${ }^{14} \mathrm{Au}$ point que les élus locaux s'inquiètent du devenir de la spécificité de la gouvernance basque au moment des travaux du comité Balladur sur la réforme territoriale de l'État en février 2009 (Vaïsse, 2009).
} 
Enjolras $^{15}$, de deux types de gouvernance : la première, verticale, centrée sur l'État et les collectivités territoriales, qui tentent ici de gérer sur un mode davantage incitatif que coercitif un territoire réfractaire ; la seconde, horizontale et fondée sur une forte dynamique locale d'autogouvernance et de coordination entre acteurs de la société civile.

Les logiques d'action des appareils politico-administratifs s'articulent ici à la demande d'une politique de la reconnaissance émanant d'une société civile mobilisée (Enjolras, 2005). Le défi est de taille pour des institutions remettant sans cesse sur le métier une démarche participative de développement reposant sur la recherche permanente du consentement. L'absence de la mainmise d'un leader politique sur l'ensemble du processus a d'ailleurs certainement favorisé la dynamique collective (Hourcade, 2007). La construction territoriale en Pays Basque français connaîtra une relance avec une nouvelle étape prospective au début des années 2000. Cette étape apparaît dans un contexte institutionnel national favorable, avec une relance de la décentralisation. L'acte II du projet de territoire, dit « Pays Basque $2020 »$, articule ainsi son programme opérationnel (Lurraldea, 2006) autour de trois chantiers prioritaires : la réciprocité territoriale entre la côte et l'intérieur du Pays Basque, la coopération transfrontalière et le développement durable. Elle ouvre du même coup de nouvelles opportunités potentielles pour les acteurs de l'ESS.

\subsection{Quel enrôlement des acteurs de l'ESS?}

La participation de l'ESS à ce tournant territorial de la gouvernance peut être appréhendée au moins sous deux angles.

On peut d'abord s'interroger sur le rôle des acteurs de l'ESS dans la genèse et le développement des nouvelles institutions. De façon formelle, d'abord, les grandes familles associatives et coopératives sont représentées au sein du Conseil de développement. De façon informelle ensuite, une sociologie plus fine des acteurs met en évidence une circulation des militants du mouvement coopératif vers les nouveaux instruments d'action publique territoriale, du moins à leur genèse. La présidence et le secrétariat général du premier Conseil de développement du Pays Basque sont ainsi attribués en 1994 à d'anciens responsables locaux et régionaux du mouvement coopératif. Ils sont les premiers experts du développement, qui, comme dans toutes les «pays », porteront le processus de traduction des intérêts localisés (Ségas 2007). Ils exercent alors une fonction de médiation entre l'offre de participation portée par la sphère politico-administrative et les intérêts et valeurs des groupes sociaux. À ce rôle moteur de certains acteurs pivots de la mouvance coopérative dans la mise sur agenda de l'institutionnalisation territoriale succède cependant une relative distanciation des acteurs de l'économie sociale vis-à-vis d'une démarche territoriale en quête d'un second souffle. Bien des organisations d'ESS ne se mobilisent que faiblement sur cette démarche, soit qu'elles disposent déjà (comme pour les associations du secteur médico-social) d'un accès routinier aux dispositifs publics par d'autres canaux institutionnels, soit, pour les structures les plus immergées dans la sphère marchande (comme les SCOP), qu'elles ne sentent pas concernées au premier chef par les mutations de l'action publique locale. Peutêtre davantage qu'une inadéquation entre action publique et société civile est-ce tout autant la difficulté d'un ensemble de secteurs et de statuts à se penser en champ unifié que cela

\footnotetext{
${ }^{15}$ Voir la contribution de Bernard Enjolras.
} 
donne à voir. Or tout le défi, pour les porteurs de la gouvernance territoriale, consiste précisément à enrôler les acteurs de l'ESS dans le processus, afin de « susciter leur adhésion, leur participation et leur implication dans une idée de construction collective des systèmes d'action publique » (Leloup, Moyart et Pecqueur, 2005, p. 329).

En opérant un renversement de perspective, on peut s'interroger sur la place des dynamiques d'ESS dans les préconisations et les démarches contractuelles issues de la démarche territoriale. C'est bien là, comme l'ont souligné Peraldi et Rombaldi (2009) pour les territoires ruraux en Corse, un enjeu de reconnaissance sociale via les décideurs publics qui se noue pour l'ESS. En Pays Basque, et à la différence de ce qui a pu être observé dans d'autres pays aquitains ${ }^{16}$, les organisations d'ESS n'apparaissent pas ici en tant que telles dans les préconisations retenues par ces travaux prospectifs davantage centrés sur les enjeux d'équilibre territorial, de structuration des activités productives en clusters ${ }^{17}$, et d'équilibre entre économie productive et économie « présentielle » (Davezies, 2004) que sur des enjeux statutaires qui valoriseraient les formules coopératives, mutualistes ou associatives. Certes, les diagnostics territoriaux dressent un panorama des défis auxquels est confronté le territoire qui sont susceptibles d'interpeller directement les acteurs de l'ESS. Le projet Pays Basque 2020 constate en effet, à côté d'un « capital territorial » fort, l'émergence de disparités territoriales, voire de fractures sociales et territoriales : disparités entre générations, entre femmes et hommes, entre niveaux de qualifications, entre quartiers et bassins de vie à cause de la concentration des emplois sur le littoral, précarisation de l'emploi, problème récurrent du foncier (maintien d'un foncier à vocation agricole et production d'un foncier à vocation économique), isolement social. Mais l'idée d'un développement local qui serait porté en grande partie par des organisations d'économie sociale et solidaire est plutôt portée aux marges des institutions de la gouvernance, notamment par le biais de la mouvance associative Hemen-Herrikoa. Sur le registre symbolique, par exemple, l'opération des « Makila ${ }^{18}$ de l'économie sociale et solidaire », lancée en 2001 par l'association Hemen en partenariat avec le Crédit coopératif et les entreprises basques, témoigne de l'ancrage du milieu associatif dans une démarche d'économie sociale, même si l'approche statutaire de l'ESS le cède ici au profit d'une approche hybride mêlant critères sociaux et territoriaux.

\footnotetext{
${ }^{16}$ Sébastien Ségas, dans une comparaison instructive entre le pays Pays Basque et le pays Médoc, montre comment le rapport des acteurs de l'économie sociale à la transformation de l'action publique peut varier selon les contextes. Dans le Médoc, les porteurs de l'action publique du pays reprennent la catégorisation statutaire du champ de l'ESS proposée par la CRESS Aquitaine, cette convergence de perspective leur donnant du même coup une forte capacité d'initiative, avec notamment l'impulsion par le pays de structures d'insertion. En Pays Basque, les dynamiques d'ESS apparaissent moins articulées en tant qu'ESS à la politique du « pays » (Ségas, 2007).

${ }^{17}$ Les travaux de Pays Basque 2020 intègrent l'approche en termes de pôles de compétitivité impulsée par l'État en 2005-2006, soit la combinaison, sur un espace donné, d'entreprises, de centres de formation et d'unités de recherche publiques ou privées engagées dans une démarche partenariale destinée à dégager des synergies autour de projets innovants. Pays Basque 2020 préconise la structuration des activités productives en clusters dans les secteurs de l'agroalimentaire, du tourisme, des bâtiments et des nouveaux matériaux, ainsi qu'un renforcement du pôle aéronautique et du pôle de compétitivité et système productif local autour des métiers de la glisse. Sans doute le recours au cluster n'est-il pas sans faire référence au Pays Basque espagnol, qui eut recours à cette formule au début des années 1990, avec un fort engagement du gouvernement autonome pour restructurer son tissu industriel. On ne trouve pas, en revanche, comme dans les travaux du CESR de Bretagne, de préconisation pour l'intégration de l'ESS au sein de clusters territoriaux d'innovation économique et sociale (Souchard, 2007).

${ }^{18}$ Bâton d'honneur.
} 


\subsection{Une illustration sectorielle : les dynamiques linguistiques et culturelles}

La faible institutionnalisation de l'ESS en tant que catégorie d'action publique en Pays Basque ne saurait cependant masquer ni la réalité des dynamiques d'action collective des acteurs associatifs et coopératifs, ni la participation de ces acteurs aux grandes tendances de la gouvernance territoriale. Discrète dans ces procédures, l'ESS fait cependant montre de sa spécificité au détour d'un secteur ou d'une mesure. On ne donnera ici qu'un exemple sectoriel, correspondant à l'un des axes privilégiés de la gouvernance territoriale : la culture et la langue basque ${ }^{19}$.

Gouverner le secteur de la culture revient en grande partie, en Pays Basque comme ailleurs, à gérer un double mouvement d'institutionnalisation et de professionnalisation d'un monde associatif aussi dynamique que diversifié. À cette problématique universelle se greffe la spécificité basque qui a fait du secteur associatif le fer de lance d'une revendication où les causes culturelle et linguistique ont été longtemps confondues. Le milieu associatif, historiquement porteur de cette mobilisation, est structuré et professionnalisé, grâce notamment à des réseaux transnationaux consolidés. Il génère en ce sens une économie culturelle, qui a expérimenté un processus d'institutionnalisation en deux temps. En 1990, c'est au sein d'un Institut culturel basque (ICB) que se fédèrent les associations culturelles, soit au sein d'une structure elle-même associative mais financée par les collectivités territoriales (syndicat intercommunal de soutien à la culture basque, département, région) et l'État. L'Institut devient dès lors en charge de la conduite d'une action publique cohérente en matière de culture basque - les autres politiques culturelles étant portées par ailleurs, notamment par des structures de la Côte basque -, à l'interface du milieu associatif, des élus et des administrations culturelles. Durant cette première phase, promotion de la culture basque et promotion de la langue basque sont confondues, même si certains segments (comme l'association Ikas) se spécialisent dans l'action pédagogique en direction du milieu scolaire. L'institutionnalisation, cependant, segmente la réalité sociale en secteurs d'action publique spécialisés, et rapidement, la nécessité d'une autonomisation de la politique linguistique à l'égard de la politique culturelle se fait ressentir. C'est chose faite au début des années 2000 , avec l'instauration d'une maîtrise d'ouvrage publique en 2001, qui débouchera en janvier 2005 sur l'Office public de la langue basque (OPLB). L'Office se charge dès lors de la réalisation des enquêtes sociolinguistiques, jusque là gérées par l'ICB pour le Pays Basque français, d'une coordination de l'enseignement du basque dans les trois réseaux scolaires (associatif immersif ikastola, public bilingue, privé-catholique bilingue ${ }^{20}$ ), et d'une coordination avec les réseaux associatifs de promotion du basque. Le secteur associatif se retrouve par là-même dans un rapport ambivalent à cette nouvelle gouvernance. Dépendants de la puissance publique quant à la pérennité de leurs financements, les associations entretiennent des rapports de participation-critique à des institutions médiatrices comme l'ICB ou l'OPLB (Oronos, 2002) (Bortayrou et al., 2005). Elles sont en tous les cas parties prenantes des nouveaux réseaux de gouvernance (Klijn, Skelcher, 2007) qui régulent désormais les relations entre acteurs publics, privés et associatifs dans ce secteur.

\footnotetext{
${ }^{19}$ Le secteur agricole constituerait un autre exemple parlant, de par l'entrelacement des débats sur l'institutionnalisation territoriale de l'agriculture basque et sur le développement de démarches d' « agriculture paysanne et durable » (Itçaina, 2009).

${ }^{20}$ Les filières d'enseignement bascophones ont leur propre dynamique d'institutionnalisation, via des contrats d'association avec le Ministère de l'Education nationale à compter du début des années 1990.
} 
L'économie générée par le secteur linguistico-culturel basque relève, à bien des égards, de l'économie sociale et solidaire. Dans un souci de stabilisation institutionnelle, plusieurs initiatives associatives semblent se tourner récemment vers le statut coopératif. Ce recours statutaire est notamment employé pour consolider des collaborations transfrontalières, rejoignant ainsi l'une des priorités de la nouvelle gouvernance territoriale. De façon significative, les fédérations des ikastola basques françaises et espagnoles décident en 2009 de constituer une société coopérative européenne. En Basse-Navarre, la télévision de pays associative Aldudarrak bideo, elle-même porteuse de plusieurs projets transfrontaliers, lance une réflexion en 2009 pour se transforme en SCIC, entendant par là-même renforcer la maîtrise des travailleurs sur leur outil de travail et sa dimension multi-partenariale. Plus directement articulée encore au tissu coopératif basque, l'association Hezkuntek se crée en 2003 avec pour objectif de favoriser l'inscription de jeunes basques français dans les cursus d'enseignement technique du Pays Basque espagnol. En effet, il s'agit pour cette association « de favoriser le développement industriel en Labourd, Basse Navarre et Soule » en promouvant « l'enseignement technique et professionnel dans les milieux bascophones ${ }^{21} »$. Ce n'est donc pas simplement l'enseignement professionnel qui est ici soutenu mais l'enseignement professionnel en langue basque ${ }^{22}$. De ce fait, Hezkuntek, outre la fédération des ikastola côté français, trouve ses partenaires sudistes naturels dans le milieu coopératif, et en particulier auprès des écoles d'ingénieurs de l'Université de Mondragón. Enfin, le projet ARIPTIC, financé par des fonds Interreg III, est porté par un Groupement Européen d'Intérêt économique (GEIE) dénommé ARINSUP, et qui regroupe deux entités : d'une part, Arizmendi Koop, coopérative de Mondragón qui développe des activités dans le domaine de l'éducation et de la formation continue à travers un réseau d'écoles faisant une large place à l'enseignement en langue basque, et intervenant également dans le champ de l'insertion sociale et professionnelle ; d'autre part, l'INSUP, organisme de formation aquitain à statut associatif qui développe depuis 1980 des activités d'insertion et de qualification professionnelle. Cette coopération débouche sur la constitution en mars 2002 d'un GEIE basé à Bayonne, première étape vers la constitution d'une société coopérative européenne. Ce partenariat s'inscrit ainsi dans une visée de promotion du modèle de l'économie sociale, auquel s'ajoute une orientation culturelle. ARINSUP a obtenu le financement par Interreg du programme ARIPTIC qui vise à expérimenter l'utilisation des technologies de l'information et de la communication dans les actions de formation (Ségas, Itçaina, 2006). Les offres d'e-formations financées par Interreg concernent aussi l'apprentissage du basque, au même titre que celui du français et de l'espagnol. La question de la formation professionnelle n'est pas ici dissociée de la promotion de l'enseignement en et $d u$ basque.

Tout se passe au fond comme si, confrontée à un double processus d'institutionnalisation et de marchandisation, le monde culturel basque cherchait dans les statuts de l'ESS, et en particulier dans le statut coopératif, une façon à la fois de renforcer la professionnalisation du secteur associatif, et un recours pour renforcer un tissu de relations transfron-

\footnotetext{
${ }^{21}$ Extraits de statuts de l'association.

${ }^{22}$ Hezkuntek reçoit en 2003 le soutien financier d'Udalbiltza, association qui regroupe des élus municipaux du Pays Basque nord et sud. En mars 2006, Hezkuntek signe une convention avec le département de l'Education du gouvernement basque autonome pour faciliter l'accès aux formations techniques professionnelles d'Euskadi aux étudiants bascophones du versant français.
} 
talières. Se renouvellent ainsi les relations horizontales qu' avaient entretenu, notamment, les mouvements coopératifs des deux côtés de la frontière dans les années 1970-1980, mais sous une forme désormais mieux articulée aux dispositifs d'action publique. Les nouvelles institutions territoriales servent alors de facilitateur pour que les projets accèdent à des dispositifs européens propices à la fois à l'institutionnalisation des relations transfrontalières (Harguindéguy, 2007) et de l'économie sociale.

\section{Conclusion}

Se joue ainsi un processus d'apprentissage des jeux d'échelle de l'action publique territoriale par les acteurs de l'ESS basque, qu'il s'agirait d'observer secteur par secteur. Même si l'ESS en tant que telle fait preuve d'une faible institutionnalisation dans la nouvelle gouvernance territoriale basque, les acteurs associatifs et coopératifs trouvent dans ce nouveau contexte d'action publique des ressources partenariales propres à renforcer leur ancrage territorial, en particulier sur le volet transfrontalier. S'actualise ainsi une mobilisation ancienne, qui doit sa genèse autant à des données contextuelles de nature culturelle et politique qu'à des facteurs économiques. L'histoire de l'économie sociale basque est indissociable de la construction sociale de la confiance territoriale, de l'émergence d'un débat public autour du développement local et des nouvelles formes de gouvernance territoriale. En ce sens, elle est un condensé de ce qu'une analyse en termes de régime territorial, accordant une place légitime aux facteurs sociopolitiques, peut apporter à l'analyse des mobilisations économiques territoriales.

\section{Références}

Azurmendi J. 1984. El hombre cooperativo. Pensamiento de Arizmendiarrieta. Caja laboral, Saint-Sébastien

Berthet T., Palard J., 1997. Culture politique réfractaire et décollage économique, l'exemple du Nord-est vendéen, Revue française de science politique 47(1), 29-47.

Bidart P. 1977. Le pouvoir politique à Baigorri, village basque, Bayonne, Ipar.

Bortayrou J., Etcheverry-Ainchart P., Garat M. et al., 2005. Le mouvement culturel basque, 1951-2001. 2. Filières bilingues, Seaska, AEK, Elkar, Bayonne, Saint-Sébastien.

Brana S., Jégourel Y. 2010. Microfinance, acteurs et territoires: analyse du secteur en Aquitaine. In Itçaina $\mathrm{X}$. (dir). La politique du lien. Les nouvelles dynamiques territoriales de l'économie sociale et solidaire. Rennes, Presses universitaires de Rennes, à paraître.

CRESS Aquitaine. 2008. Les chiffres clés de l'économie sociale et solidaire en Aquitaine, Bordeaux, CRESS Aquitaine.

Chaussier J.-D., 1996. Quel territoire pour le Pays Basque? Les cartes de l'identité. L'Harmattan, Paris.

Demoustier D., 2001. L'économie sociale et solidaire. S'associer pour entreprendre autrement. Syros, La Découverte, Paris.

Dupuy C., Torre A. 2004. Confiance et proximité. In Pecqueur B., Zimmermann J.-B. (dir.). Economie de proximité. Paris, Hermes, Lavoisier, 65-87.

Enjolras B., 2005. Le nouveau discours normatif sur la société civile. Cahiers de l'ARUC-ES, n $14-2005,1-20$.

Evers A., 2001. The significance of social capital in the multiple goals and resource structure of social enterprises. in Borzaga C., Defourny J. (ed.), The emergence of social enterprise, Routledge, London, New York, 296-311. 
Harguindéguy J.-B. 2007. Cross-border policy in Europe. Implementing INTERREG III-A France-Spain, Regional and Federal Studies, 17(3), 317-334.

Herria (1967), «Eztuinagako laborarien kooperatifaz, berriz ere », Herria, 8 juin.

Hourcade R., 2007. Le Pays Basque en représentations, ou les effets sur l'action publique et le territoire d'un instrument de "gouvernance » locale : le Conseil de développement du Pays Basque. Master recherche Action et Espaces Publics en Europe. Institut d'Etudes Politiques de Rennes, Rennes.

Itçaina, X., 2007a. Des entreprises politiques ? Les SCOP dans l'espace public basque, in Itçaina X., Palard J., Ségas S. (dir.), Régimes territoriaux et développement économique, Presses universitaires de Rennes, Rennes, 67-82.

Itçaina X., 2007b. Les virtuoses de l'identité. Religion et politique en Pays Basque. Presses universitaires de Rennes, Rennes.

Itçaina X. et Palard J., 2007. Introduction : culture, territoire, systèmes productifs. In Itçaina X., Palard J., Ségas S. (dir.), Régimes territoriaux et développement économique, Presses universitaires de Rennes, Rennes, 11-24.

Itçaina, X.. 2009. «La représentation agricole en débat : le cas du Pays Basque français », Economie rurale, $\mathrm{n}^{\circ} 312$, p. 52-65.

Klijn E.-H., Skelcher C. 2007. Democracy and governance networks: compatible or not? Public administration, 85(3), 587-608.

Lagroye J., 1997. Sociologie politique. FNSP, Dalloz, Paris.

Lagroye J., 2003. Les processus de politisation. In Lagroye J. (dir.) La politisation. Belin, Paris, 359-372.

Lascoumes P., Le Galès P., 2007. Sociologie de l'action publique. Armand Colin, Paris.

Leloup F., Moyart L. Pecqueur B., 2005. La gouvernance territoriale comme nouveau mode de coordination territoriale ? Géographie, économie, société. 7(4), 321-332.

Lung Y., 2007. Vers une méso-économie politique du territoire ? in Itçaina J., Palard J., Ségas S. (dir.), Régimes territoriaux et développement économique. Presses universitaires de Rennes, Rennes, 313-318.

Lurraldea (2006), Pays Basque 2020 : Acte II du projet de territoire, Programme opérationnel, validé par l'assemblée plénière du 9 décembre.

Newton K., 1999. Social capital and democracy in modern Europe. in Van Deth J. W., Maraffi M., Newton K. \& Whiteley P. F. (eds.) Social capital and European democracy. Routledge, London, New York, 3-24.

Oronos M. 2002. Le mouvement culturel basque, 1951-2001, t. 1. Ikas, Pizkundea, Euskal konfederazioa, Bayonne, Saint-Sébastien, Elkar.

Palard J., 2009. La Beauce inc. Capital social et capitalisme régional. Montréal, Les Presses de l'Université de Montréal.

Peraldi X., Rombaldi M. 2009. La contribution de l'économie sociale et solidaire au développement rural: quelques constats dans le contexte corse. Géographie, économie société. 11(2), 161-179.

Reix F. 2008. L'ancrage territorial des créateurs d'entreprises aquitains : entre encastrement relationnel et attachement symbolique. Géographie, économie, société. 10(1), 29-41.

Ritaine E., 2001. Cherche capital social, désespérément. Critique internationale. 12, 48-59.

Ségas S., 2004. La grammaire du territoire : action publique de développement et lutte politique dans les «pays». Thèse de doctorat en science politique. Université de Bordeaux IV, Pessac.

Ségas S., 2007. Le détour de l'action publique : enthousiasme et déception des acteurs de l'économie sociale face aux politiques de développement territorial. In Itçaina X., Palard J., Ségas S. (dir.). Régimes territoriaux et développement économique. Presses universitaires de Rennes, Rennes, 247-260.

Ségas S., Itçaina X., 2006. L'économie sociale et solidaire et les territoires du politique : une comparaison franco-espagnole. Colloque international Économie sociale et solidaire en Europe : quel avenir ?, Institut d'Etudes Politiques de Grenoble, 1-2 juin 2006.

Simoulin V., 2007. La gouvernance territoriale : dynamiques discursives, stratégiques et organisationnelles. In Simoulin V., Weisbein J. (dir.). La gouvernance territoriale. Pratiques, discours et théories. Droit et société n44, L.G.D.J., Paris, 15-32. 
Souchard N., 2007. L'économie sociale et solidaire en région Bretagne : traditions coopératives et contrastes des dynamiques contemporaines. In Itçaina X. (dir.) Les territoires de l'économie sociale et solidaire: vers une comparaison interrégionale européenne. Rapport de recherche, programme de recherche DIIESES, Spirit, Sciences Po Bordeaux, Pessac, 53-81.

Stone D. A., 1989. Causal stories and the formation of policy agendas. Political science quarterly, 104(2), 281-300.

Trigilia C., 2002. Sociologie économique. État, marché et société dans le capitalisme moderne. Armand Colin, Paris. Vaïsse S., 2009. Rapport Balladur : les élus locaux réagissent déjà. Le Journal du Pays Basque, 26 février. 
1 